\title{
Chaos Detection of Rule Weak Periodic Signal
}

\author{
Guihua Yang ${ }^{1, a}$ 、 Junchi Ma ${ }^{1, b} 、$ Yukai Gao ${ }^{1, c} 、$ Chuanying Wang ${ }^{1}$ \\ ${ }^{1}$ Department of Physics and Electrical Information Engineering,Daqing Normal University,Daqing, \\ Heilongjiang, 163712, China \\ âstillwatersss@163.com, bhorseman_1111@163.com, gaoyukai@hit.edu.cn
}

\section{Keywords: Chaos;Weak Periodic Signal;Detection}

Abstract: In the various scientific and technological fields, because of the kinds of interference, more commonly measured signal is a periodic signal which is often more complex waveforms, to detect such a signal having the necessity and practical significance. They can not be used to describe a simple mathematical functions, it can exist under colored noise background in any distribution. So we find a chaos detection system capable of detecting rule periodic signal.

\section{Chaos Detection System Model of periodic signal}

When constructing a sinusoidal signal detection model, use the characteristics of system which is extremely sensitive to changes in the built-in signal amplitude, place a test signal with the same frequency of signal detection system for internal chaos detection system built-in signal. Adjusted the built-in signal amplitude to the critical value before joining the test signal and with the addition of the test signal ,measures amplitude variation with minimal impact force generated by the magnitude of the state will make the system a fundamental change, that is the system state from chaos into a cycle of large-scale state. This change can be observed through the simulation graphics, strong observability, this is the reason to choose this change as a signal detection based.But the above ideas can not be used to construct a built-in signal,Therefore, a method ofjoin periodic signal to suppress the use of chaos is generate. This method was added to the chaotic system periodic perturbation, in order to suppress the chaos, no prior knowledge of the signal waveform to be measured, simply add a weak periodic perturbation in the coefficient item's original Duffing equation $x^{3}$, the equation becomes

$$
b\left[1+\eta \cos \left(\omega_{1} t\right)\right] x^{3}=-\delta x+\gamma \cos \left(\omega_{2} t\right)
$$

Where $\eta \cos \left(\omega_{1} t\right)$ is $x^{3}$ 'scoefficient, as system's perturbation; $\eta(<<1)$ is amplitude of parameter perturbation ; $\gamma \cos (\omega t)$ is system's built-in signal ; $\omega_{1}$ is perturbation frequency。

In the absence of the parameter perturbation, which is $\eta=0$, the system is in chaotic attractor state, after $x^{3}$, s coefficient plus a small period perturbation,can be converted to the desired cycle state, that state is suppressed by chaos. This has been demonstrated by Melnikov method and numerical methods.After a large number of simulation experiments, taking into account the sensitivity of the basis of the stability of the test signal on two aspects of the system, increasing the nonlinear term $x^{5}$, periodic perturbation factor into coefficients $x^{5}$ items. Mathematical model of the system by the following formula:

$$
k \ll-x^{3}+[1+a s(\omega t)] x^{5}=\gamma \sin (\omega t)
$$

Where as $(\omega t)$ is test cycle signal ; $\gamma \sin (\omega t)$ is built-in signal。

From the signal theory shows that when any periodic signal $a s(\omega t)$ satisfy Dirichlet condition, which can develop into a triangular form of the Fourier series, which is $a s(\omega t)=a_{0}+\sum_{n=1}^{\infty}\left(a_{n} \cos n \omega t+b_{n} \sin n \omega t\right)$.

Equation (2) is converted into the following (3) in the form:

$$
k x=\gamma \sin (\omega t)-[1+a s(\omega t)] x^{5}+x^{3}
$$


White noise signal white noise signal generator through the amplifier with any periodic signal obtained by Fourier expansion of two trigonometric signals and external constant input signal of constant adder 1 to the sum 3, and then multiply it fifth power coefficient has been required perturbation signal by adder 2 will be $+k x$.

Then equation (3) into (4) form, by the adder 1 obtained After two integral role in both integrator to obtain the value of $x$, and time-domain waveform and phase plane trajectory of $x$ calculated.

$$
\gamma \sin (\omega t)-[1+a s(\omega t)] x^{5}+x^{3}-k \&
$$

Model shown in Fig 1.

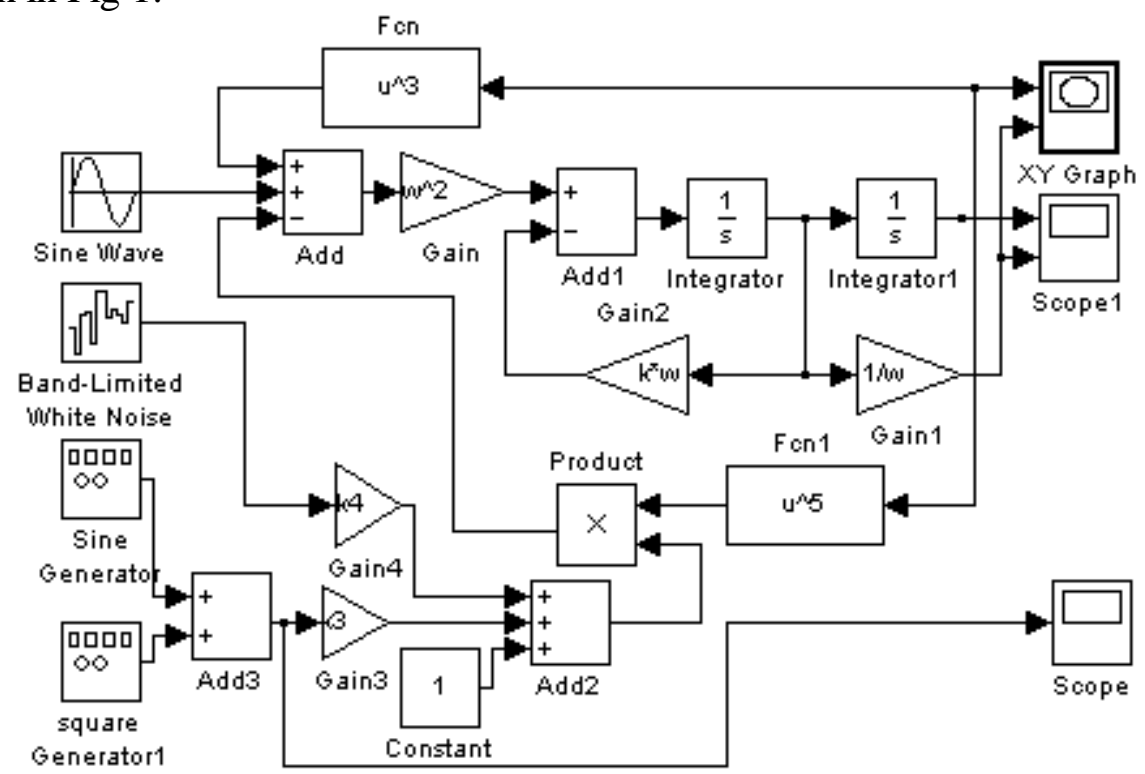

Fig.1 The system model with weak periodic perturbation

\section{Chaotic systems detection rules periodic signal and dynamic behavior}

First, we will set measured small signal $a s(\omega t)$ amplitude $a$ to 0 , and added noise power is $10^{-4} \mathrm{~W}$ band-limited white noise. $\gamma$ to observe the changes in the system state by changing the built-in signal amplitude $\gamma \cos (\omega t)$.

When $\gamma=0.65$,the system is in a chaotic state. Time-domain waveform output is random, the output of the phase space is random curve, the time-domain waveform and corresponding phase space trajectory as shown in Figure 2.

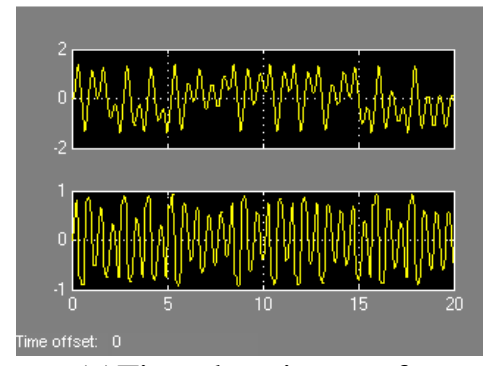

(a)Time-domain waveform

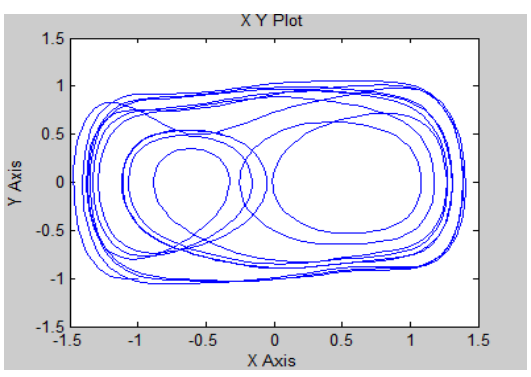

(b)Phase plane trajectory

Fig.2 The system is in the chaotic state while $\gamma=0.65$

When $\gamma=0.72698980$,the system is in a critical state ravioli. When this state ravioli state transition to large-scale periodic state of critical state, will be very sensitive to external signals, the system time-domain waveform and phase plane trajectories shown in Figure 3. 


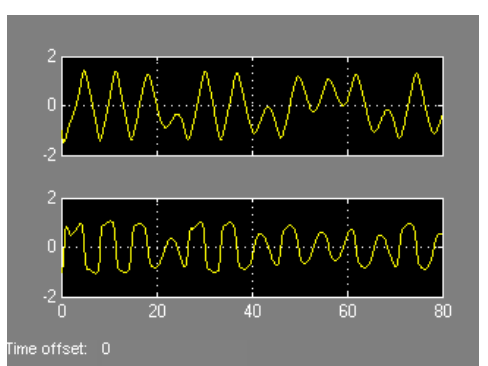

(a)Time-domain waveform

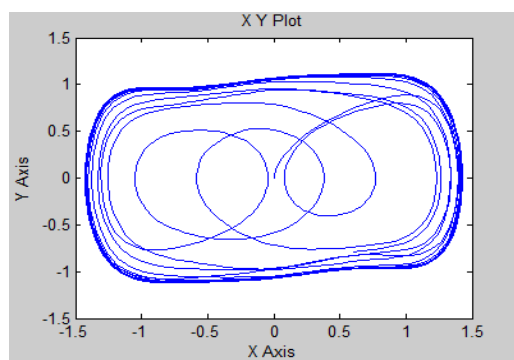

(b)Phase plane trajectory

Fig.3 The system is in the critical chaotic state while $\gamma=0.72698980$

When $\gamma=0.72698981$, the system is in the large-scale periodic status field output is a periodic waveform. Its phase trajectory is a single orbit around the two centers closed curve, as shown in the phase plane trajectories and the corresponding time-domain waveform in Figure 4.

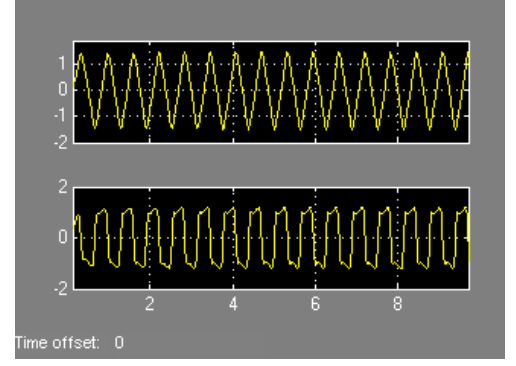

(a)Time-domain waveform

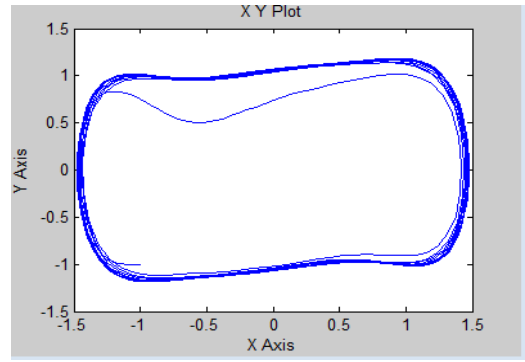

(b)Phase plane trajectory

Fig.4 The system is in the large-scale periodic state while $\gamma=0.72698981$

Adjusted the system to a critical state, and even if the built-in signal amplitude $\gamma=0.72698980$, then small signal added to the system under test. Adjusting the amplitude of the signal being measured, can be found as long as the amplitude of the test signal is greater than a critical value, the system status will be changed by the critical state of the large-scale periodic state.

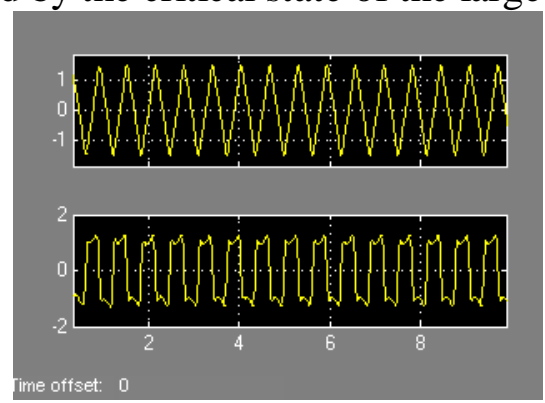

(a)Time-domain waveform

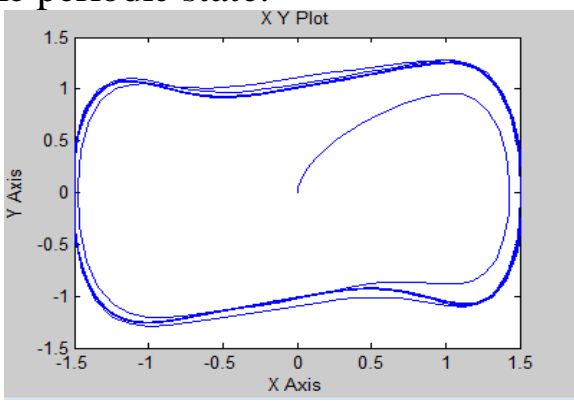

(b)Phase plane trajectory

Fig.5 The system is in more stable large-scale periodic state while $\gamma=0.8$

In the critical state after the system was added as a small perturbation of the measured signal by the chaotic state of the system becomes large-scale periodic state, indicating that the added periodic perturbation reached suppression system chaos purposes. The values obtained from the simulation can be seen, the system in the critical state of the system is very sensitive to changes in parameters, system parameters very small perturbation will cause a fundamental change in the system state. When the cycle perturbation amplitude is $10^{-7} \mathrm{~V}$ magnitude, the system state is changed, the detection accuracy is satisfactory.

Chaotic systems have immunity to noise simulation and experimental analysis, this immunity refers primarily when the system is in a chaotic state, the influence of this state from noise, and then a big can not change, chaos or chaos. But when the noise added to the system increases, the disorder of the system will increase. Need to increase the degree of order in the system to overcome the effects of this disorder, can the system from a high degree of disorder of the chaotic state of the large-scale transition to an ordered state cycle. Increase the degree of order to the threshold cause the system to change the status of the corresponding period of large-scale increase, so that the system state shift cycle 
perturbation lowest amplitude signal is also increased accordingly. Noise is too large, the detection performance of the system will be severely degraded.

Any periodic signal detection model and the sinusoidal signal detection model compared to the same basic form can know that although the two models, but when the same noise into the system, the system is not the same as the threshold where lower threshold sinusoidal signal detection model . By equation (3) shows small-signal and noise test cycle together as perturbation $x^{5}$ coefficient, while the disorder of the system, the nonlinear perturbation of the noise of the same size, the impact on the system is big. Simulation set noise power to a large value $10^{-4} \mathrm{~W}$, the resulting cycle perturbation minimum amplitude of the signal, which is the lowest detection limit system for $10^{-7} \mathrm{~V}$, So the minimum detection threshold of the chaotic system is limited to: $10 \lg \left(4 \times 10^{-7}\right)=-64(d B)$. At the same time, after a lot of simulation experiments, the system can be measured SNR minimum threshold for the work:

$$
S N R=10 \lg \longrightarrow=10 \lg \left[\frac{0.5\left(4 \times 10^{-7}\right)^{2}}{1 \times 10^{-4}}\right]=-91(\mathrm{~dB})
$$

Currently it handles time-domain approach signal to noise ratio of the minimum threshold only $-10 d B$, compared with the time domain method to detect weak signals, the nonlinear chaotic oscillator cycle in weak signal detection, this method has high sensitivity, intuitive, simple, easy to implement, has a relatively low signal to noise ratio and other characteristics limit work .

\section{conclusion}

Non-linear restoring force term improvement Duffing equation, build a new model of weak periodic signal detection, the specific methods of chaos theory to detect weak periodic signal is discussed, analyzed Duffing added additional perturbation form, and based on this form established a mathematical model and corresponding computer simulation model, the simulation model, a series of simulation experiments verify the feasibility of the system, the model can achieve weak periodic signal detection under strong noise background, to verify the relatively traditional time domain detection SNR door method limit is much lower, improving the detection signal to noise ratio, improve the detection accuracy, and certainly the detection capabilities of the system.

\section{Acknowledgements}

This work was financially supported by The Education Department of Heilongjiang province science and technology research projects (12543004)

\section{References}

[1] Shang Qiufeng, Yin Chengqun , Li Shilin. Study on detection of weak simusoidal signal by using doffing oscilator[J]. Zhongguo Dianji Gongcheng Xuebao, 2005, 25: 66-70.

[2] Suykens J, Vandewalle J. Generation of $n$-double scrolls $(n=1,2,3,4)$ [J].IEEE Trans . Circuits Syst. (Part-I), 1993, 40(11): $861 \sim 867$.

[3] Yalcin M E, Suykens J, Vandewalle J. Experimental confirmation of 3-and 5-scroll attractors from a generalized Chua's circuit[J]. IEEE Trans.Circuits Syst. (part-I), 2000, 47(3): 425 429.

[4] Guihua Yang,Junchi Ma,Yukai Gao.Achieve Positioning of Embedded NLOS Mobile Robot[J],Applied Mechanics and MaterialsVols.687-691(2014):105-108. 